\title{
Association of Thyroid Stimulating Hormone with Insulin Resistance in Women with Polycystic Ovarian Syndrome
}

\author{
Naher $\mathrm{S}^{1}$, Begum $\mathrm{SR}^{2}$, Ali $\mathrm{L}^{3}$, Hakim $\mathrm{M}^{4}$
}

\begin{abstract}
Introduction: Polycystic Ovarian Syndrome (PCOS) is a major cause of female infertility which is thought to be associated with Insulin Resistance (IR). However the nature and degree of IR have been shown to vary in different populations. PCOS women have also been reported to have increased prevalence of subclinical hypothyroidism which has been postulated to be determinants of IR. The natures of thyroid involvement have also been shown to vary between populations.
\end{abstract}

Objectives: The study was undertaken to explore the distribution of IR and thyroid dysfunction as assessed by Thyroid Stimulating Hormone (TSH) levels among PCOS subjects and also to investigate the association of IR with TSH in women with PCOS.

Materials and Methods: One hundred and fifty one PCOS patients (age in years $24 \pm 5$; $M \pm S D$ ) were studied. PCOS was diagnosed by Rotterdam criteria. Fasting serum C-peptide was measured by Enzyme Linked Immuno Sorbide Essay (ELISA) and serum TSH was measured by Microparticle Enzyme Immunoassay (MEIA). Serum glucose was estimated by Glucose-Oxidase method (GOD-PAP). Insulin sensitivity was assessed by using Homeostasis Model Assessment (HOMA).

Results: The mean serum C-peptide (nmol/l) and HOMA $\%$ S were $0.67 \quad( \pm 0.35)$ and $85 \quad( \pm 42)$ respectively. The median (Range) serum $\mathrm{TSH}$ level $(\mu \mathrm{l} \mathrm{U} / \mathrm{ml})$ of the study subjects was found to be 2.49 (0.66 to 20.86). Insulin sensitivity was found to be $26 \%, 47 \%, 17 \%$ and $10 \%$ in those who had HOMA\%S level at the range of $<50,50-100,101-150$ and $>150$ respectively. Of the total PCOS subjects, $85 \%$ had normal level of TSH value whereas only 15\% PCOS subjects had subclinical hypothyroidism. The median serum TSH level of the insulin resistance and non-resistance groups were $2.25(0.89-5.71)$ and 2.58 (0.74-20.86) respectively. On Pearson's correlation analysis insulin sensitivity was not found to be any significant association with $\mathrm{TSH}$ in the PCOS subjects.

Conclusion: The study revealed that there was no significant association with IR and TSH in the PCOS subjects.

Key-words: Thyroid Stimulating Hormone, Insulin Resistance, Polycystic Ovarian Syndrome.

\section{Introduction}

Polycystic Ovarian Syndrome (PCOS) is the most common endocrine disorder in women ${ }^{1}$. It generates numerous health problems and it is usually common at puberty. It affects $4-12 \%$ women of reproductive age $^{2}$. Despite being heterogeneous in nature, the hallmarks of the disease are hyperandrogenism and chronic anovulation. In a view of an increased prevalence of PCOS in Caribbean Hispanics, it has been suggested that there might be ethnic variation in overt features and biochemical abnormalities of $\mathrm{PCOS}^{3}$. Of the community studies conducted so far to determine the prevalence of polycystic ovaries, the highest reported prevalence was $52 \%$ among South Asian Immigrants in Britain ${ }^{4}$. Reaven cited that after described by Stein and Leventhai in 1935 much has been learned about the patho-physiology of PCOS

1. Dr. Salma Naher, MBBS, MS, FCPS (Obs \& Gynae), Junior Consultant, Kurmitola General Hospital, Dhaka;

2. Prof Sultana Rajia Begum, MBBS, FCPS, Ex Professor of Obstetrics \& Gynaecology, BSMMU, Dhaka;

3. Prof Liaquat Ali, MBBS, PhD, Professor of Biochemistry and Cell Biology, Director, BIHS, Coordinator, Biomedical Research Group, BIRDEM, Dhaka; 4. Lt Col Maksumul Hakim, MBBS, MPH, MPhil, Associate Professor of Community Medicine, AFMC. 
from its neuroendocrine underpinnings ${ }^{5}$ to an ever growing understanding of the link between obesity, IR and PCOS ${ }^{6}$. In women with PCOS, approximately $50-70 \%$ has been reported to have hyperinsulinemic $\mathrm{IR}^{7}$ and metabolic syndrome which increase the risk for type 2 diabetes mellitus and cardiovascular diseases ${ }^{8}$. IR is defined where a normal or elevated insulin level produces an attenuated biological response. Classically this refers to impaired sensitivity to insulin mediated glucose disposal. Several groups have reported that women with PCOS are more IR then would be expected on the basis of their age and body mass index (BMI) ${ }^{9}$. The mechanisms underlying this phenomenon are not fully understood. Although hyperandrogenemia may play a role ${ }^{10,11}$ other factors such as thyroid function may also be involved.

Little is known about the relationship between IR and thyroid function. Thyroid dysfunction is common in women of reproductive age, with a prevalence of elevated TSH ranging from $4-9 \%$ in this population ${ }^{12}$. Recently there has been increasing interest in the relationship between thyroid function, weight and metabolic status.

Hypothyroidism is associated with obesity, dyslipidemia and increased atherosclerotic vascular disease $^{13}$. A similar cluster consisting of obesity, dyslipidemia, diabetes and hypertension was proposed by Reaven (1998) under the name Metabolic Syndrome (MS). MS also leads to atherosclerotic vascular diseases and IR is the central pathophysiological phenomenon underlying this clustering ${ }^{14}$. Women with PCOS have a high prevalence of increased TSH level with frequent prevalence of concomitent IR and MS. Subclinical hypothyroidism developing in addition may aggravate IR and other risk factors.

However, Jackson et al (1970) studied the OGTT, insulin response to glucose and glucose disposal per unit of insulin; although IR after $50 \mathrm{~g}$ of oral glucose was more in hypothyroidism before treatment, glucose disposal per unit of insulin was not decreased in the hypothyroid state, arguing against the presence of IR in hypothyroidism. Some other studies also suggested that thyroid disturbance does not enhance the IR and hyperinsulinemia $^{15,16}$. Women with PCOS might therefore be candidates for screening, in order to identify any disturbances in thyroid function and any changes in metabolic parameters, as well as IR indices. In the above context the present study was undertaken to estimate the frequency distribution of IR and TSH levels among PCOS subjects, and to explore the association of IR and TSH in this disorder.

\section{Materials and Methods}

This is a cross-sectional comparative study which was undertaken in Bangladesh Institute of Research and Rehabilitation in Diabetes, Endocrine and Metabolic Disorders (BIRDEM) and Department of Gynecology \& Obstetrics, Bangabandhu Sheikh Mujib Medical University (BSMMU). The study duration was January 2009 to December 2010. One hundred and fifty one Bangladeshi PCOS women aged between 15-40 years were included in this study. PCOS was diagnosed by the presence of two out of the following three criteria (The Rotterdam Eshre/Asrm, 2004):

\section{$\Rightarrow$ Oligomenorrhoea and/ or anovulation \\ $\Rightarrow$ Hyperandrogenism \\ $\Rightarrow$ Polycystic ovaries}

\section{-Exclusion Criteria \\ $\Rightarrow$ PCOS women already known to be diabetic \\ $\Rightarrow$ PCOS with other endocrinopathy like hyper- adrenalism and hypothyroidism.}

For each and every subject a separate data collection sheet was prepared. Subjects were selected by a purposive sampling procedure according to the availability of the patients who fulfilled the inclusion criteria. Sample size calculation and blood collection were taken under the following procedure. Since the ultimate test of association between IR and TSH or BMI in this study was multiple regression analysis, the sample size was calculated based on the number of independent variables. The simplified method, as described by Mascie Taylor and Rahman was used. In this case $\mathrm{n}$ were $>104+m$, where $m$ is the number of independent variables for testing the multiple correlations. With IR as dependent variable and $\mathrm{TSH}, \mathrm{BMI}$ and age as independent variables, we have a small sample size $\geq 104+1, \geq 105$, For better power of the study we included 150 PCOS subjects. Whole venous blood was taken from the subject 
following an overnight fasting (12hr) in the follicular phase of a spontaneous or progestin induced cycle in women who were not on any form of medication. Blood sample from each subject was taken by a $5 \mathrm{cc}$ disposable syringe. After 30 minutes of blood drawing, serum was separated by centrifugation at $3000 \mathrm{rpm}$ for $10 \mathrm{~min}$ and then the serum was collected at least $600 \mu \mathrm{l}$ in each tube (4). Blood samples were maintained at $4^{\circ} \mathrm{C}$ until separation and plasma was frozen at $-20^{\circ} \mathrm{C}$ within an hour of sample collection. Fasting plasma glucose, insulin \& TSH were assayed by appropriate procedure. Serum was not allowed to be thawed until the assay is performed.

$\Rightarrow$ Glycemic status of the study subjects was measured by OGTT, using Glucose Oxidase method (Randox, UK).

$\Rightarrow \mathrm{TSH}$ was measured by Chemiluminescent Immunometric Assay (Immulite, USA).

$\Rightarrow$ Serum C-peptide was measured by ELISA method (Linco Research Ltd, USA).

$\Rightarrow \mathrm{IR}$ was calculated by fasting glucose and C-peptide using HOMA using HOMA Software Calculator-2.

- Operational definitions

$\Rightarrow$ Serum TSH $(\mu \mathrm{IU} / \mathrm{ml})$ level were divided into lower normal and higher (subclinical hypothyroidism) with median (2.49) value as the cut-off point.

$\Rightarrow$ Insulin sensitivity (HOMA \%S) was divided into insulin resistant and insulin nonresistant groups with $50 \% \mathrm{HOMA} \% \mathrm{~S}$ as the cut-off point.

\section{Statistical analysis}

Data were compiled in pre-designed case record forms and expressed as $\mathrm{M} \pm S D$ or Median (range) as applicable. In case of continuous variable, difference between groups was explored by using Student's t-test (normally distributed data) or Mann-Whitney Test (for nonparametric distribution). Simple correlation between two groups was analyzed by Pearson's correlation test. Association between IR and TSH was further investigated by multivariate analysis with IR as dependent variables and TSH and other relevant clinical, anthropometric or biochemical factors as independent variables. In all cases $p<0.05$ was considered as significant. SPSS software for windows version 12.0 was used to analysis the data.

\section{Results}

In the present study, 151 PCOS women were included. All the subjects were within the reproductive age (15-40 years) group.

Insulin sensitivity (HOMA \%S) was assessed by HOMA. The mean $( \pm S D)$ serum C-peptide $(\mathrm{nmol} / \mathrm{l})$ and HOMA \%S were $0.67( \pm 0.35)$ and $85( \pm 42)$ respectively. The median (Range) serum thyroid stimulating hormone $(\mathrm{TSH})$ level $(\mu \mathrm{lU} / \mathrm{ml})$ of the study subjects was found to be 2.49 (0.66 to 20.86) (Table-I).

Table-I: Insulinemic and TSH status of the study subjects $(n=151)$.

\begin{tabular}{|l|l|}
\hline Parameter & Mean \pm SD $/$ Median (Range) \\
\hline C-peptide $(\mathrm{nmol} / \mathrm{l})$ & $0.67 \pm 0.35$ \\
\hline HOMA $\%$ S & $85 \pm 42$ \\
\hline TSH $(\mu \mathrm{IU} / \mathrm{ml})$ & $2.49(0.66-20.86)$ \\
\hline
\end{tabular}

Forty seven $(47 \%)$ percent subjects were in the range of 50 to 100 of insulin sensitivity whereas, $26.5 \%$ were lower insulin sensitivity. In addition $16.6 \%$ and $9.9 \%$ were in the range of 101 to 150 and $>150$ respectively (Figure-1).

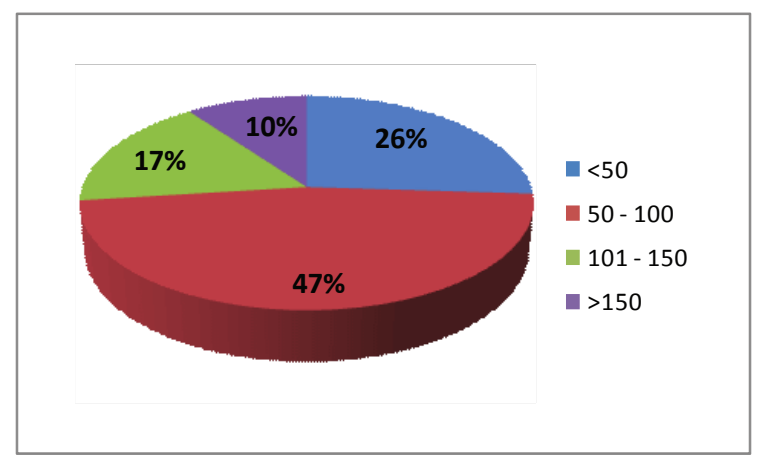

Fig-1: Frequency distribution of insulin sensitivity among PCOS subjects.

About fifteen (15\%) percent subjects showed TSH value $>4.50 \mu \mathrm{lU} / \mathrm{ml}$ whereas $85 \%$ PCOS subjects showed TSH values within 0.40 to $4.50 \mu \mathrm{l} \cdot \mathrm{U} / \mathrm{ml}$ range (Figure-2):

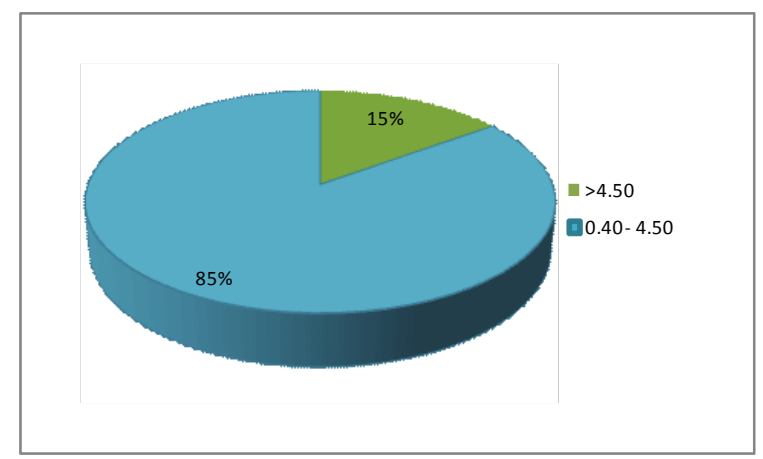

Fig-2: Frequency distribution of TSH among PCOS subjects. 
The median (Range) TSH level $(\mu \mathrm{lU} / \mathrm{ml}$ ) of the IR and non-resistance groups were $2.25(0.89-5.71)$ and 2.58 (0.74-20.86) respectively (Table-II).

Table-II: TSH status among insulin resistance and non-resistance group of PCOS subjects.

\begin{tabular}{|c|c|c|}
\hline Parameter & $\begin{array}{c}\text { Insulin resistance } \\
\text { Median (Range) }(\mathrm{n}=40)\end{array}$ & $\begin{array}{c}\text { Insulin non-resistance } \\
\text { Median(Range) }(\mathrm{n}=111)\end{array}$ \\
\hline TSH $(\mu \mathrm{l} \mathrm{U} / \mathrm{ml})$ & $2.25(0.89-5.71)$ & $2.58(0.74-20.86)$ \\
\hline
\end{tabular}

Results were expressed as Median (range), n-number of subjects

There was no correlation between insulin sensitivity and TSH among the study subjects $(r=0.081$, $\mathrm{p}=0.323)(\mathrm{Fig}-3)$.

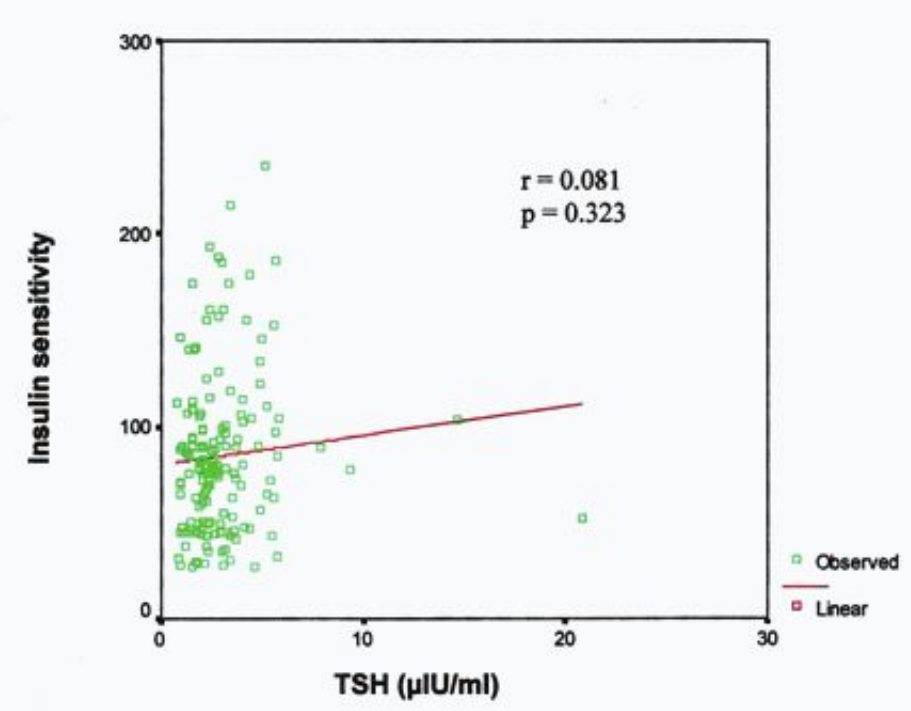

Fig-3: Correlation between insulin sensitivity and TSH in PCOS subjects.

\section{Discussion}

PCOS is now considered as a disorder of the MS, which increases the risk of Type-2 diabetes mellitus and cardiovascular diseases substantially ${ }^{17}$. Like many other disorders of MS, IR is thought to be a central phenomenon where many of the other abnormalities converge. The increased prevalence of IR in PCOS is widely acknowledged ${ }^{18}$, but the exact degree of IR has been reported to vary from population to population. In a study by Deugarte et al (2005) found that the value of HOMA\%S was $64.4 \%$ in USA PCOS population. In another study on Canadian population ${ }^{19}$, the value was $75 \%$.

The PCOS subjects in the present study were fairly young $(24 \pm 5$ years, $M \pm S D)$. The mean $( \pm S D)$ of HOMA\%S in the present population was $85( \pm 42)$ which indicate that IR in Bangladeshi PCOS subjects may be substantially less than that of USA or Canadian population. Taken as a whole only $26 \%$ of PCOS subjects were found to have HOMA \%S below 50 which means that only one-fourth of the PCOS subjects in our population have severe IR.

The etiopathogenesis of IR in PCOS subjects is a matter of further controversy since multiple primary and secondary factors seem to be involved in this case. Apart from the yet undetected possible primary factors, hyperadrogenemia ${ }^{11}$ seems to be a major contributing factor in the genesis of IR in PCOS. However, factors like age, BMI and often-antagonistic hormones also seem to play a major contributing or confounding role in this case.

Thyroid hormones are one of the most important insulin antagonists and those have been already investigated in PCOS. The frequency distribution of TSH levels (Fig-2) showed a clear pattern of thyroid function status in the PCOS subjects. Whereas $85 \%$ of subjects showed normal TSH values $(0.4-4.5$ $\mu \mathrm{lU} / \mathrm{ml}), 15 \%$ showed levels compatible with subclinical hypothyroidism ( $>4.5 \mu \mathrm{lU} / \mathrm{ml})$. Janssen et al (2004) reported similar proportion of subclinical hypothyroidism in PCOS among European subjects. In a cross sectional study of South India where the proportion of subclinical hypothyroidism ${ }^{20}$ was found to be $20 \%$.

HOMA-IR is an reliable yet inexpensive and empirical mathematical formula based on fasting glucose and fasting insulin levels that was developed as a surrogate measurement of clinical diabetes research to measure insulin sensitivity ${ }^{21,22}$. In the present study, insulin sensitively measured by this method showed no significant difference of TSH level between insulin resistant and non-resistant groups. There are reports that hypothyroidism, even in the subclinical stage, is associated with $\mathrm{IR}^{23,24}$. However, Ganie et al (2011) reported that subclinical hypothyroidism is not associated with alteration in phenotypic expression and IR in young women with PCOS. This is in contrast to the findings of Gang et al (2010) who found significant negative correlation between TSH and HOMA-IR in PCOS subjects. In this study, there was lack of association between insulin sensitivity and TSH reflected in the lack of significant correlation between HOMA\%S and TSH in PCOS. The present data is not consistent with 
authors ${ }^{25,26}$ who found an association of IR with subclinical hypothyroidism in PCOS. However, in a recent study on Indian population Ganie et al (2011) found no association of subclinical hypothyroidism with IR in young women with PCOS. Another study by Brenta et al (2007) also found no association of insulin sensitivity as measured by HOMA-IR and QUICKI with subclinical hypothyroidism. It seems that TSH may not play a crucial role in the development of IR in PCOS.

\section{Conclusion}

Only about one-fourth subjects with PCOS have severe IR. About $15 \%$ of PCOS subjects suffer from subclinical hypothyroidism. Thyroid function abnormality seems to have no association with IR in PCOS.

\section{References}

1. Fenichel P, Gobert B, Carre $Y$ et al. Polycystic ovary syndrome in autoimmune disease. Lancet 1999; 353: 140-6736.

2. Knochenhauer ES, Key TJ, Kahsar-MIller M et al. Prevalence of the polycystic ovary syndrome in unselected black and white women of the south eastern United States: a prospective study. J Clin Endocrinol Metab 1998; 83: 3078-82.

3. Solomons CG. The epidemiology of Polycystic Ovary Syndrome prevalence and associated disease risks. Endocrinol \& Metab Clin N Am 1999; 28: 247-63.

4. Rodin DA, Bano G, Bland JM et al. Polycystic ovaries and associated metabolic abnormalities in Indian subcontinent Asian women. Clin Endocrinol 1998; 49: 91-9.

5. Rebar R, Judd HL, Yen SS et al. Characterization of the inappropriate gonadotropin secretion in polycystic ovarian syndrome. J Clin Invest 1976; 57: 1320-9.

6. Gambineri A, Pelusi C, Vicennati V et al. Obesity and the polycystic ovary syndrome. Int J obes Relat Metab Disord 2002; 26: 883-96.

7. Dunaif A. Insulin resistance and the polycystic ovary syndrome: mechanism and implications for pathogenesis. Endo Rev 1997; 18: $774-800$

8. Azziz R, Woods KS, Reyna R et al. The prevalence and features of the polycystic ovary syndrome in an unselected population. J Clin Endocrinol Metab 2004; 89: 2745-9.

9. Barber TM, McCarthy MI, Wass JA et al. Obesity and polycystic ovarian syndrome. Clin Endocrinol (Oxf) 2006; 65-437-45.

10. Mogohetti $P$, Tosi $F$, Castello $R$ et al. The insulin resistance in women with hyper androgenism is partially reversed by antiandrogen treatment evidence that androgens impair insulin action in women. J Clin Endocrinol Metab 1996; 81: 952-60.
11. Mohlig $M$, Jurgens $A$, Spranger $J$ et al. The androgen receptor CAG repeat modifies the impact of testosterone on insulin resistance in women with Polycystic Ovary Syndrome, Eur $\mathrm{J}$ Endocrinol 2006; 155: 127-30.

12. Canaris GJ, Manowits NR, Mayor G et al. The Colorado thyroid disease prevalence study. Arch Int Med 2000; 28: 526-34.

13. Coppola AR, Ladenson PW. Hyperthyroidism and atherosclerosis. J Clin Endocrine/ Metab 2003; 88: 2438-44.

14. Eckel RH, Grundy SM, Zimmet PZ. The Metabolic syndrome. Lancet 2005; 365: 1415-28.

15. Brenta G, Berg G, Arias P et al. Lipoprotein alterations, hepatic lipase activity, and insulin sensitivity in subclinical hypothyroidism: response to L-T4 treatment. Thyroid 2007; 17:453-60.

16. Ganie MA, Laway BA, Wani TA et al. Association of subclinical hypo- thyroidism and phenotype, insulin resistance, and lipid para- meters in young women with polycystic ovary syndrome. Fertil Steril 2011; 95: 2039-43.

17. Teede HJ, Hutchison S, Zoungas $\mathrm{S}$ et al. Insulin resistance, the metabolic syndrome, diabetes, and cardiovascular disease risk in women with PCOS. Endrocrine 2006; 30: 45-53.

18. Hart R. Polycystic ovarian syndrome prognosis and treatment outcomes. Obs\&Gyne 2007; 19: 529-35.

19. Kieren J, Verma S, Corenblum B et al. Normal Endothelial Function Despite Insulin Resistance in Healthy Women with the Polycystic Ovary Syndrome. J Clin Endocrinol Metab 2000; 85: 1851-6.

20. Shantha PS, Kumar A, Jeyachandran V et al. Association between primary hypothyroidism and metabolic syndrome and the role of $C$ reactive protein: A cross-sectional study from South India. Thyroid Research 2009; 2: 1-7.

21. Hermans et al. Comparison of insulin sensitivity tests across a range of glucose tolerance from normal to diabetes. Diabetologia 1999; 42: 678-87

22. Song $Y$, Manson $E$, Tinker $L$ et al. Insulin Sensitivity and Insulin Secretion Determined by Homeostasis Model Assessment (HOMA) and Risk of Diabetes in a Multiethnic Cohort of Women: The Women's Health Initiative Observational Study. Diabetes Care 2007; 30: 1747-52.

23. Tuzcu A, Bahceci M, Gokalp D et al. Subclinical hypothyroidism may be associated with elevated high-sensitive c-reactive protein (low grade inflammation) and fasting hyperinsulinemia. J Endocrinol 2005; 52: 89-94.

24. Dessein PH, Joffe BI, Stanwix AE. Subclinicai hypothyroidism is associated with insulin resistance in rheumatoid arthritis. Thyroid 2004; 14: 443-6.

25. Mueller A, Scho C, Dittrich $\mathrm{R}$ et al. Thyroid-stimulating hormone is associated with insulin resistance independently of body rnass index and age in women with polycystic ovary syndrome. Hum Reprod 2009; 24:2924-30.

26. Dittrich $\mathrm{R}$, Kajaia N, Cupisti $S$ et al. Association of thyroid stimulating hormone with insulin resistance and androgen parameters in women with PCOS. Report Biomed Online 2009; 19: 319-25. 